TRANSACTIONS OF THE

AMERICAN MATHEMATICAL SOCIETY

Volume 354, Number 1, Pages 47-61

S 0002-9947(01)02818-5

Article electronically published on August 21, 2001

\title{
COHOMOLOGY OF BUILDINGS AND FINITENESS PROPERTIES OF $\widetilde{A}_{n}$-GROUPS
}

\author{
JACQUI RAMAGGE AND WAYNE W. WHEELER
}

\begin{abstract}
Borel and Serre calculated the cohomology of the building associated to a reductive group and used the result to deduce that torsion-free $S$-arithmetic groups are duality groups. By replacing their group-theoretic arguments with proofs relying only upon the geometry of buildings, we show that Borel and Serre's approach can be modified to calculate the cohomology of any locally finite affine building. As an application we show that any finitely presented $\widetilde{A}_{n}$-group is a virtual duality group. A number of other finiteness conditions for $\widetilde{A}_{n}$-groups are also established.
\end{abstract}

\section{INTRODUCTION}

An important result of Borel and Serre shows that a torsion-free $S$-arithmetic group $\Gamma$ is a duality group [BS]. In other words, there exist a natural number $d$ and a $\mathbb{Z} \Gamma$-module $D$ such that for every integer $i$ there is a natural isomorphism

$$
H^{i}(\Gamma,-) \cong H_{d-i}(\Gamma, D \otimes-) \text {. }
$$

Borel and Serre obtain this result by first studying the building associated to a reductive group $G$. In particular, they calculate the Alexander-Spanier cohomology of the building and deduce that a torsion-free $S$-arithmetic subgroup of $G$ is a duality group.

The purpose of this paper is to show that the ideas of Borel and Serre, which seem to depend upon properties of the group $G$, can be adapted so that they apply to other situations as well. In particular, we calculate the cohomology of an arbitrary locally finite affine building $\Delta$. Since $\Delta$ need not arise from an associated reductive group, the arguments given here are necessarily more geometric than those used by Borel and Serre. Our hope is that an approach relying only upon the geometry of buildings will be accessible to a wider audience than one relying upon the properties of reductive groups. At the same time, the ideas presented here should help to make it clearer which arguments in [BS] depend fundamentally upon the group $G$ and which arguments depend only upon properties of the associated building.

As an application of the result describing the cohomology of $\Delta$, we study the homological finiteness properties of certain groups acting on buildings. Groups that act simply transitively on the vertices of a thick $\widetilde{A}_{2}$-building in a type-rotating manner, known as $\widetilde{A}_{2}$-groups, were introduced in [CMSZ1] and [CMSZ2. The more general notion of an $\widetilde{A}_{n}$-group was studied in [C]. Any such group has a

Received by the editors March 29, 2000.

2000 Mathematics Subject Classification. Primary 13D25, 20 J06.

(C)2001 American Mathematical Society 
relatively simple presentation with respect to which the Cayley graph is the 1skeleton of an $\widetilde{A}_{n}$-building. One of the reasons why such groups are of interest is that for $n=2$ they give rise to buildings that cannot be obtained from classical groups. We show that any finitely generated $\widetilde{A}_{n}$-group $\Gamma$ is a virtual duality group; in other words, $\Gamma$ has a subgroup $K$ of finite index that is a duality group. The argument proving this result applies uniformly to all $\widetilde{A}_{n}$-groups, but a shorter proof is possible in some cases. For $n>2$ we show that the result can be obtained as a consequence of the work in [BS]; for $n=2$ we include an argument of G. Robertson using Kazhdan's Property (T) to prove that certain $\widetilde{A}_{2}$-groups are virtual duality groups.

The broad outline of the paper generally follows the proof of Borel and Serre that torsion-free $S$-arithmetic groups are duality groups. In particular, Section 3 constructs a compact space $\bar{X}$ in which the locally finite affine building $\Delta$ embeds as an open subspace. Section 4 develops geometric arguments to calculate the Alexander-Spanier cohomology with compact supports $H_{c}^{*}(\Delta ; \mathbb{Z})$. In Section 5 the cohomology $H_{c}^{*}(\Delta ; \mathbb{Z})$ is used to show that finitely presented $\widetilde{A}_{n}$-groups are virtual duality groups, and a number of other finiteness conditions are also given.

The authors are indebted to John Crisp for helpful discussions on the material in Section 3. The work presented here was partially supported by the University of Newcastle and the University of Leicester, and the second author was partially supported by the National Science Foundation. The authors are grateful to all of these organizations for their assistance.

\section{BACKGROUND MATERIAL}

The purpose of this section is to summarize some definitions, properties, and notational conventions for buildings and for Alexander-Spanier cohomology that are used in the remainder of the paper. The primary references for buildings are [B2] and $[\mathrm{R}]$; a good reference for Alexander-Spanier cohomology is $[\mathbf{S}$.

Recall that a building $(\Delta, \mathcal{A})$ is a simplicial complex $\Delta$ and a collection $\mathcal{A}$ of subcomplexes called apartments satisfies the following axioms:

(1) $\Delta=\bigcup_{A \in \mathcal{A}} A$

(2) every apartment $A \in \mathcal{A}$ is a Coxeter complex;

(3) for any two simplices $\sigma_{1}, \sigma_{2} \in \Delta$ there is an apartment $A$ containing both $\sigma_{1}$ and $\sigma_{2}$

(4) if $A$ and $A^{\prime}$ are two apartments containing simplices $\sigma_{1}$ and $\sigma_{2}$, then there is an isomorphism $A \rightarrow A^{\prime}$ fixing $\sigma_{1}$ and $\sigma_{2}$ pointwise.

The conventions used throughout this paper are generally the same as those used in [B2]. In particular, we regard chambers as open subsets of the geometric realization of $\Delta$. Because we are usually more concerned with the geometric realization of $\Delta$ than with $\Delta$ itself, we have chosen also to write $\Delta$ for the geometric realization of the building when no confusion is likely to arise.

If $\Delta$ is a building, then the apartments of $\Delta$ are all isomorphic. For any apartment $A$ there is a Coxeter group $(W, S)$ such that $A$ is isomorphic to the Coxeter complex $\Sigma(W, S)$, and this Coxeter group gives a corresponding $W$-valued distance function on the set of chambers of $A$. If $\mathcal{C}$ is the set of chambers of $\Delta$, then this function extends to a $W$-valued distance function $\delta: \mathcal{C} \times \mathcal{C} \rightarrow W$. When $W$ is finite, the building $\Delta$ is said to be spherical; when $W$ is infinite and irreducible but 
the subgroup generated by any proper subset of $S$ is finite, $\Delta$ is said to be affine. The terminology in these cases reflects the structure of the geometric realizations of apartments, which are homeomorphic to either $n$-spheres or affine $n$-space for some fixed $n$. Other types of buildings play no part in the work presented here and will not be considered.

An affine building $\Delta$ is a path-metric space with unique geodesics. Moreover, $\Delta$ satisfies the CAT(0)-property. In other words, if $x, y \in \Delta$ and $0 \leq t \leq 1$, then the point $z=(1-t) x+t y$ on the geodesic $[x, y]$ satisfies the inequality

$$
d(w, z)^{2} \leq(1-t) d(w, x)^{2}+t d(w, y)^{2}-t(1-t) d(x, y)^{2}
$$

for all $w \in \Delta$ (see Lecture 4 of [B3] $)$.

If $\Delta$ is an affine building, then a standard construction produces another building $\Delta_{\infty}$, called the spherical building at infinity. A ray in $\Delta$ is a subset $\mathfrak{r}$ that is isometric to the half-line $[0, \infty)$; the points of $\Delta_{\infty}$ are the equivalence classes of rays under translation. Let $x_{0}$ be a special vertex in $\Delta$. Then the points in $\Delta_{\infty}$ are in one-toone correspondence with the rays based at $x_{0}$, and the chambers in $\Delta_{\infty}$ correspond to sectors based at $x_{0}$.

Throughout this work we write simply $H^{i}(X ; \mathbb{Z})$ for the $i$ th Alexander-Spanier cohomology group of a space $X$ because that is the only cohomology theory used; similarly, we write $H_{c}^{i}(X ; \mathbb{Z})$ for the $i$ th Alexander-Spanier cohomology group of $X$ with compact supports, and $\tilde{H}^{i}(X ; \mathbb{Z})$ denotes the reduced $i$ th Alexander-Spanier cohomology group of $X$. In particular, $H^{0}(X ; \mathbb{Z})$ is isomorphic to the group of locally constant functions from $X$ to $\mathbb{Z}$, and $H_{c}^{0}(X ; \mathbb{Z})$ is isomorphic to the subgroup of locally constant functions with compact supports. The reduced Alexander-Spanier cohomology agrees with the ordinary Alexander-Spanier cohomology in positive degrees and satisfies $\tilde{H}^{0}(X ; \mathbb{Z})=0$ if $X$ is connnected. We use a number of properties of Alexander-Spanier cohomology in the following sections, and the reader is encouraged to consult $\underline{\underline{S}}]$ or $[\mathrm{Ma}]$ for further details.

\section{An analogue of the Borel-Serre space}

In [BS] Borel and Serre studied the building $\Delta$ of a reductive group by constructing a compact space $\bar{X}$ and a homeomorphism $\phi$ from $\Delta$ onto an open subspace of $\bar{X}$ such that the points of $X_{\infty}=\bar{X}-\phi(\Delta)$ are in one-to-one correspondence with those of the building at infinity $\Delta_{\infty}$. The purpose of this section is to construct and study the space $\bar{X}$ when $\Delta$ is an arbitrary locally finite affine building. It is necessary, therefore, to replace the group-theoretic arguments of Borel and Serre by geometric proofs. These proofs rely heavily upon the fact that $\Delta$ is a complete metric space. But the constructions of this section work somewhat more generally: it is only necessary to assume that $\Delta$ is a complete, locally compact CAT(0)-space with a metric $d$, and we will work in this more general setting throughout this section.

Fix a point $x_{0} \in \Delta$. Let $X$ denote the collection of all geodesics $\left[x_{0}, x\right]$ for $x \in \Delta$, and let $X_{\infty}$ denote the collection of all geodesic rays $\left[x_{0}, e\right)$ based at $x_{0}$. A geodesic $\left[x_{0}, x\right]$ of length $s$ can be regarded as a function $\gamma_{x}:[0, \infty) \rightarrow \Delta$ that is an isometry on $[0, s]$ and is constant on $[s, \infty)$; similarly, a geodesic ray $\left[x_{0}, e\right)$ can be regarded as an isometry on its entire domain. Thus $X$ and $X_{\infty}$ are disjoint subsets of the collection $C([0, \infty), \Delta)$ of continuous functions from $[0, \infty)$ to $\Delta$. The topology of compact convergence on $C([0, \infty), \Delta)$ then gives the subspace $\bar{X}=X \cup X_{\infty}$ a 
topological structure. If $K \subseteq[0, \infty)$ is compact, $\gamma:[0, \infty) \rightarrow \Delta$ is in $\bar{X}$, and $\varepsilon>0$, then the set

$$
B_{K}(\gamma, \varepsilon)=\left\{\gamma^{\prime} \in \bar{X} \mid d\left(\gamma(t), \gamma^{\prime}(t)\right)<\varepsilon \text { for all } t \in K\right\}
$$

is open in $\bar{X}$. In fact, the sets of this form are a basis for the topology on $\bar{X}$. By using the properties of $\mathrm{CAT}(0)$-spaces, however, one can simplify this description with the following lemma.

Lemma 3.1. Let $\Delta$ be a complete, locally compact CAT(0)-space. Suppose that $y, z \in \Delta$ are distinct points such that $d\left(x_{0}, y\right)=d\left(x_{0}, z\right)>0$, and assume that $y^{\prime} \in\left[x_{0}, y\right]$ and $z^{\prime} \in\left[x_{0}, z\right]$ satisfy $d\left(x_{0}, y^{\prime}\right)=d\left(x_{0}, z^{\prime}\right)$. Then $d\left(y^{\prime}, z^{\prime}\right) \leq d(y, z)$; equality holds if and only if $y^{\prime}=y$ and $z^{\prime}=z$.

Proof. Let $s=d\left(x_{0}, y\right)=d\left(x_{0}, z\right)$ and $s^{\prime}=d\left(x_{0}, y^{\prime}\right)=d\left(x_{0}, z^{\prime}\right)$. Then $t=s^{\prime} / s$ satisfies $0 \leq t \leq 1$; moreover, $y^{\prime}=(1-t) x_{0}+t y$ and $z^{\prime}=(1-t) x_{0}+t z$. The CAT(0)-inequality implies that

$$
d\left(y^{\prime}, z^{\prime}\right)^{2} \leq(1-t) d\left(y^{\prime}, x_{0}\right)^{2}+t d\left(y^{\prime}, z\right)^{2}-t(1-t) d\left(x_{0}, z\right)^{2}
$$

and that

$$
d\left(z, y^{\prime}\right)^{2} \leq(1-t) d\left(z, x_{0}\right)^{2}+t d(z, y)^{2}-t(1-t) d\left(x_{0}, y\right)^{2} .
$$

Hence

$$
d\left(y^{\prime}, z^{\prime}\right)^{2} \leq(1-t)\left(s^{\prime}\right)^{2}+t^{2} d(y, z)^{2}-t^{2}(1-t) s^{2}=t^{2} d(y, z)^{2} .
$$

Thus $d\left(y^{\prime}, z^{\prime}\right) \leq t d(y, z) \leq d(y, z)$, and $d\left(y^{\prime}, z^{\prime}\right)=d(y, z)$ if and only if $t=1$. The desired result now follows immediately.

To apply Lemma 3.1, consider a basic open set $B_{K}(\gamma, \varepsilon)$ in $\bar{X}$. Because $K$ is a compact subset of $[0, \infty)$, it contains a largest element $M$. Then $K \subseteq[0, M]$, and Lemma 3.1 implies that $B_{K}(\gamma, \varepsilon)=B_{[0, M]}(\gamma, \varepsilon)$. We can therefore simplify the notation by writing $B_{M}(\gamma, \varepsilon)$ for $B_{[0, M]}(\gamma, \varepsilon)$, and the sets $B_{M}(\gamma, \varepsilon)$ with $\gamma \in \bar{X}$ and $M$ and $\varepsilon$ positive form a basis for the topology on $\bar{X}$.

Lemma 3.2. Let $\Delta$ be a complete, locally compact $\mathrm{CAT}(0)$-space, and let $S$ be a subset of $\Delta$. Then $S$ is compact if and only if it is closed and bounded.

Proof. Fix $x \in S$, and set $B_{r}(x)=\{y \in \Delta \mid d(x, y)<r\}$. Then $\left\{B_{r}(x) \cap S \mid r>0\right\}$ is an open cover of $S$. If $S$ is compact, then it follows that $S \subseteq B_{r}(x)$ for some $r>0$, so $S$ is bounded. Because $\Delta$ is Hausdorff, $S$ is also closed.

Conversely, suppose that $S$ is closed and bounded in $\Delta$. Choose $r>0$ such that $S \subseteq B_{r}(x)$. Then the closure $\bar{B}_{r}(x)$ is compact by Proposition 2.3 of [P], so $S$ is also compact, as desired.

For $r>0$ set $B_{r}=\left\{x \in \Delta \mid d\left(x_{0}, x\right)<r\right\}$. Let $\bar{B}_{r}$ be the closure of $B_{r}$ in $\Delta$, and set $\partial \bar{B}_{r}=\bar{B}_{r}-B_{r}$. Let $\phi: \Delta \rightarrow \bar{X}$ be the function given by $\phi(x)=\left[x_{0}, x\right]$. It is easy to check that $\phi$ is a homeomorphism of $\Delta$ onto $X$, so we can regard $\Delta$ as an open subspace of $\bar{X}$. Moreover, the subspace $X_{\infty} \subseteq \bar{X}$ is compact by Ascoli's Theorem (Theorem 7.6.1 of [Mu]).

Proposition 3.3. Let $\Delta$ be a complete, locally compact CAT(0)-space. Then the space $\bar{X}$ is compact. 
Proof. It suffices to show that if $\mathcal{U}$ is a covering of $\bar{X}$ by basic open sets, then $\mathcal{U}$ has a finite subcover. Because $X_{\infty}$ is compact, there are open sets $U_{1}, \ldots, U_{k} \in \mathcal{U}$ such that $\left\{U_{1} \cap X_{\infty}, \ldots, U_{k} \cap X_{\infty}\right\}$ is an open cover of $X_{\infty}$. For $1 \leq i \leq k$ write $U_{i}=B_{r_{i}}\left(\gamma_{i}, \varepsilon_{i}\right)$ for some $\gamma_{i} \in \bar{X}$ and some positive numbers $r_{i}$ and $\varepsilon_{i}$. Set $r=\max \left\{r_{1}, \ldots, r_{k}\right\}$, and suppose that $x \in \Delta$ satisfies $d\left(x_{0}, x\right)=s>r$. The geodesic $\left[x_{0}, x\right]$ in $\Delta$ is the initial segment of some ray $\left[x_{0}, e\right)$ in $X_{\infty}$, and the choice of $U_{1}, \ldots, U_{k}$ implies that there is an $i$ with $\left[x_{0}, e\right) \in U_{i} \cap X_{\infty}$. Then $\phi(x)=\left[x_{0}, x\right] \in U_{i}$, and we conclude that

$$
\bar{X}-\left(U_{1} \cup \cdots \cup U_{k}\right) \subseteq \phi\left(\bar{B}_{r}\right) .
$$

But $\phi\left(\bar{B}_{r}\right)$ is compact by Lemma 3.2 so there are open sets $U_{k+1}, \ldots, U_{m} \in \mathcal{U}$ such that $\phi\left(\bar{B}_{r}\right) \subseteq U_{k+1} \cup \cdots \cup U_{m}$. Then $\left\{U_{1}, \ldots, U_{m}\right\}$ is a finite subcover of $\bar{X}$, and $\bar{X}$ is compact. This completes the proof.

Theorem 3.4. Let $\Delta$ be a complete, locally compact CAT(0)-space. Then the topology $\mathcal{T}$ on $\bar{X}$ has the following properties:

(1) the space $\bar{X}$ is compact and contractible;

(2) $X$ is open and $X_{\infty}$ is closed in $\bar{X}$;

(3) $\Delta$ is homeomorphic to $X$.

Proof. Proposition 3.3 shows that $\bar{X}$ is compact. To see that it is contractible, suppose that $\gamma \in \bar{X}$ and $t \in[0,1]$. Let $\gamma_{t} \in \bar{X}$ be the geodesic satisfying the following conditions:

(a) if $\gamma=\phi(x)$ for some $x \in \Delta$ and $x_{t} \in \Delta$ is the unique point on the geodesic $\gamma$ with $d\left(x_{0}, x_{t}\right)=\frac{t d\left(x_{0}, x\right)}{1+(1-t) d\left(x_{0}, x\right)}$, then $\gamma_{t}=\left[x_{0}, x_{t}\right]$

(b) if $\gamma \in X_{\infty}$ and $t<1$, then $\gamma_{t}=\left[x_{0}, x_{t}\right]$ for the unique point $x_{t}$ on the geodesic $\gamma$ satisfying $d\left(x_{0}, x_{t}\right)=\frac{t}{1-t}$

(c) if $\gamma \in X_{\infty}$ and $t=1$, then $\gamma_{t}=\gamma$.

Define $F: \bar{X} \times[0,1] \rightarrow \bar{X}$ by setting $F(\gamma, t)=\gamma_{1-t}$. Then $F$ is continuous and is a contraction of $\bar{X}$ onto $\gamma_{0}=\left[x_{0}, x_{0}\right]$, so (1) holds. The remaining statements follow easily from the definition of $\mathcal{T}$, and this completes the proof.

\section{The cohomology of AN AfFine BUilding}

If $\Delta$ is a locally finite affine building, then the previous section describes a compact space $\bar{X}$ such that the points of $X_{\infty}$ are canonically identified with those of the building $\Delta_{\infty}$. In this section we calculate $H^{*}\left(X_{\infty} ; \mathbb{Z}\right)$ and use the result to determine the cohomology $H_{c}^{*}(\Delta ; \mathbb{Z})$. The arguments are analogous to those used by Borel and Serre $\mathrm{BS}$, who studied the building associated to a reductive group. By avoiding their group-theoretic arguments, however, we obtain a more general result that verifies an assertion of Brown in Lecture 5 of B3].

Throughout this section let $\Delta$ be a locally finite affine building. It will be convenient to assume that the fixed base point $x_{0} \in \Delta$ is a special vertex. Then the collection $\mathfrak{C}$ of all chambers in $\Delta_{\infty}$ can be identified with the set $\mathcal{S}$ of open sectors in $\Delta$ based at $x_{0}$. If $\mathfrak{c}_{1}, \mathfrak{c}_{2} \in \mathfrak{C}$ are chambers represented by sectors $\sigma_{1}, \sigma_{2} \in \mathcal{S}$, then there are unique chambers $C_{1} \subseteq \sigma_{1}$ and $C_{2} \subseteq \sigma_{2}$ of $\Delta$ with $x_{0} \in \bar{C}_{1} \cap \bar{C}_{2}$; moreover, if $\mathfrak{c}_{1}$ and $\mathfrak{c}_{2}$ are distinct and adjacent, then so are $C_{1}$ and $C_{2}$, and in this case the adjacency type of $C_{1}$ and $C_{2}$ determines that of $\mathfrak{c}_{1}$ and $\mathfrak{c}_{2}$. 
Although $X_{\infty}$ and $\Delta_{\infty}$ are equal as sets, the topology on $X_{\infty}$ is not the usual simplicial topology on $\Delta_{\infty}$ in which a set is open if and only if its intersection with every closed simplex is relatively open. For example, if $\Delta$ is a tree, then $\Delta_{\infty}$ is a discrete space, whereas $X_{\infty}$ is compact by Theorem 3.4. Thus $X_{\infty}$ and $\Delta_{\infty}$ must be different topological spaces if $\Delta$ has infinitely many ends. The fact that the topologies on $X_{\infty}$ and on $\Delta_{\infty}$ can differ presents a minor technical problem: if $\mathfrak{c}$ is a chamber of $\Delta_{\infty}$ and $\overline{\mathfrak{c}}$ is its closure in $\Delta_{\infty}$, then it is perhaps not obvious that $\overline{\mathfrak{c}}$ is also closed in $X_{\infty}$. We begin by proving this result.

Proposition 4.1. Let $\mathfrak{c}$ be a chamber in $\Delta_{\infty}$, and let $\overline{\mathfrak{c}}$ be its closure in $\Delta_{\infty}$. Then $\overline{\mathfrak{c}}$ is also closed in $X_{\infty}$.

Proof. Let $\sigma \in \mathcal{S}$ be the sector representing $\mathfrak{c}$, and let $\bar{\sigma}$ be the closure of $\sigma$ in $\Delta$. Then $\overline{\mathfrak{c}}$ is just the collection of all geodesic rays based at $x_{0}$ that are contained in $\bar{\sigma}$. Thus if $\left[x_{0}, e\right) \in X_{\infty}-\overline{\mathfrak{c}}$, then there is a point $y \in\left[x_{0}, e\right)-\bar{\sigma}$. Choose $\varepsilon>0$ such that the open ball in $\Delta$ with radius $\varepsilon$ and center $y$ does not intersect $\bar{\sigma}$, and set $r=d\left(x_{0}, y\right)$. Then $B_{r}\left(\left[x_{0}, e\right), \varepsilon\right)$ is an open neighborhood of $\left[x_{0}, e\right)$ in $\bar{X}$ that does not intersect $\overline{\mathfrak{c}}$. Hence $\overline{\mathfrak{c}}$ is closed in $X_{\infty}$, as desired.

If $\mathfrak{c}$ is a chamber in $\Delta_{\infty}$, then Proposition 4.1 implies that its closure in $\Delta_{\infty}$ is the same as its closure in $X_{\infty}$. Thus we can refer unambiguously to its closure $\overline{\mathbf{c}}$.

Now fix an apartment $A_{0}$ in $\Delta$ containing $x_{0}$, and let $\mathfrak{a}_{0}$ be the corresponding apartment in $\Delta_{\infty}$. Then there is a finite Coxeter group $(W, S)$ such that $\mathfrak{a}_{0}$ is isomorphic to the Coxeter complex of $(W, S)$. If $w \in W$, let $\ell(w)$ be the length of $w$. Write $W=\left\{w_{1}, \ldots, w_{N}\right\}$, where the indices are chosen so that $\ell\left(w_{i}\right) \leq \ell\left(w_{j}\right)$ whenever $i \leq j$. In particular, $w_{1}=1$. For $1 \leq m \leq N$ let

$$
I_{m}=\left\{s \in S \mid \ell\left(w_{m} s\right)>\ell\left(w_{m}\right)\right\} .
$$

Then $I_{1}=S, I_{N}=\emptyset$, and $I_{m}$ is a nonempty proper subset of $S$ for $1<m<N$. Let $\delta$ be the $W$-valued distance function defined on the set of chambers of $\Delta_{\infty}$, and let $\mathfrak{c}_{0}$ be a fixed chamber in $\mathfrak{a}_{0}$. For each element $w_{i} \in W$ let $\mathfrak{C}_{i}$ be the set of all chambers $\mathfrak{c}$ in $\Delta_{\infty}$ such that $\delta\left(\mathfrak{c}_{0}, \mathfrak{c}\right)=w_{i}$, and let $X_{m}$ be the union of closed chambers defined by

$$
X_{m}=\bigcup_{i=1}^{m} \bigcup_{\mathfrak{c} \in \mathfrak{C}_{i}} \overline{\mathfrak{c}}
$$

Then $\overline{\mathfrak{c}}_{0}=X_{1} \subseteq X_{2} \subseteq \cdots \subseteq X_{N}=X_{\infty}$. Set

$$
L_{m}=\bigcup_{I \subseteq I_{m}}\left(\mathfrak{c}_{0}\right)_{I}
$$

the union of the $I$-residues of $\mathfrak{c}_{0}$. The set $L_{m}$ is open in $\overline{\mathfrak{c}}_{0}$. Indeed, the complement $L_{m}^{\prime}=\overline{\mathfrak{c}}_{0}-L_{m}$ is the union of the closures of the faces $\left(\mathfrak{c}_{0}\right)_{\{s\}}$ of codimension one for $s \in S-I_{m}$.

Example 4.2. We illustrate the above definitions when $X_{\infty}$ is the building of $\mathrm{PGL}_{3}\left(\mathbb{F}_{2}\right)$, pictured below with a distinguished chamber $\mathfrak{c}_{0}$. In this case $X_{\infty}$ is the building at infinity for a suitable affine building $\Delta$ that is neither thick nor homogeneous. 


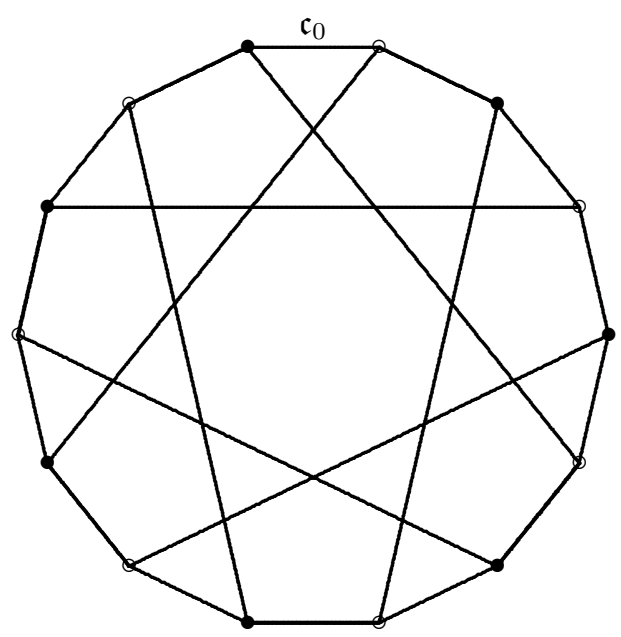

The Coxeter group $W$ is a symmetric group on three letters; it has a generating set $S=\left\{s_{1}, s_{2}\right\}$, where $s_{i}$ denotes the transposition $(i, i+1)$. The solid nodes in the diagram represent $s_{1}$-adjacency, and the circles represent $s_{2}$-adjacency. We order the elements of $W$ by setting

$w_{1}=1, \quad w_{2}=s_{1}, \quad w_{3}=s_{2}, \quad w_{4}=s_{1} s_{2}, \quad w_{5}=s_{2} s_{1}, \quad w_{6}=s_{1} s_{2} s_{1}=s_{2} s_{1} s_{2}$.

Then

$$
\begin{gathered}
I_{1}=\left\{s_{1}, s_{2}\right\}, \quad I_{2}=I_{5}=\left\{s_{2}\right\}, \quad I_{3}=I_{4}=\left\{s_{1}\right\}, \quad I_{6}=\emptyset, \\
L_{1}=\bullet, \quad L_{2}=L_{5}=\longrightarrow, \quad L_{3}=L_{4}=\bullet, \text { and } L_{6}=
\end{gathered}
$$

Moreover, $\mathfrak{C}_{1}=\left\{\mathfrak{c}_{0}\right\}$, and in the following diagrams the solid lines represent the chambers that are elements of the set $\mathfrak{C}_{i}$ for $2 \leq i \leq 6$.
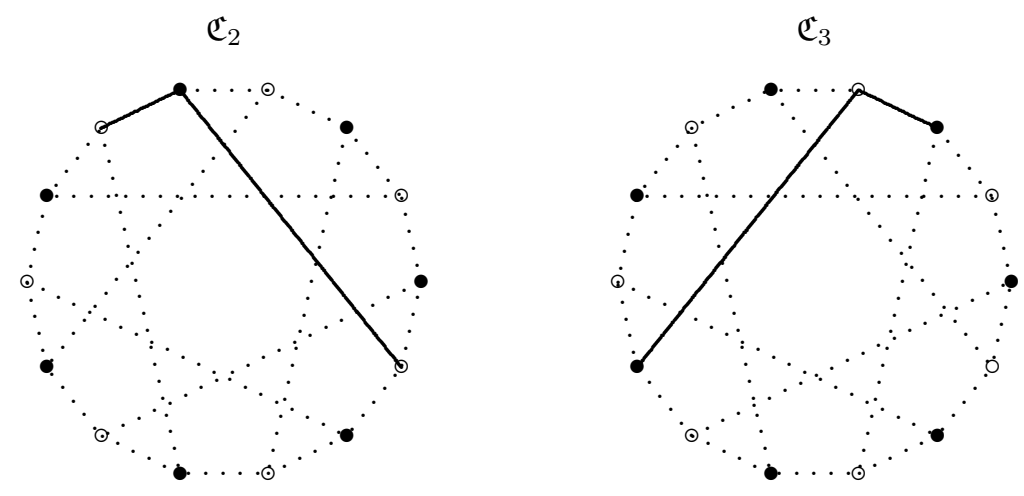

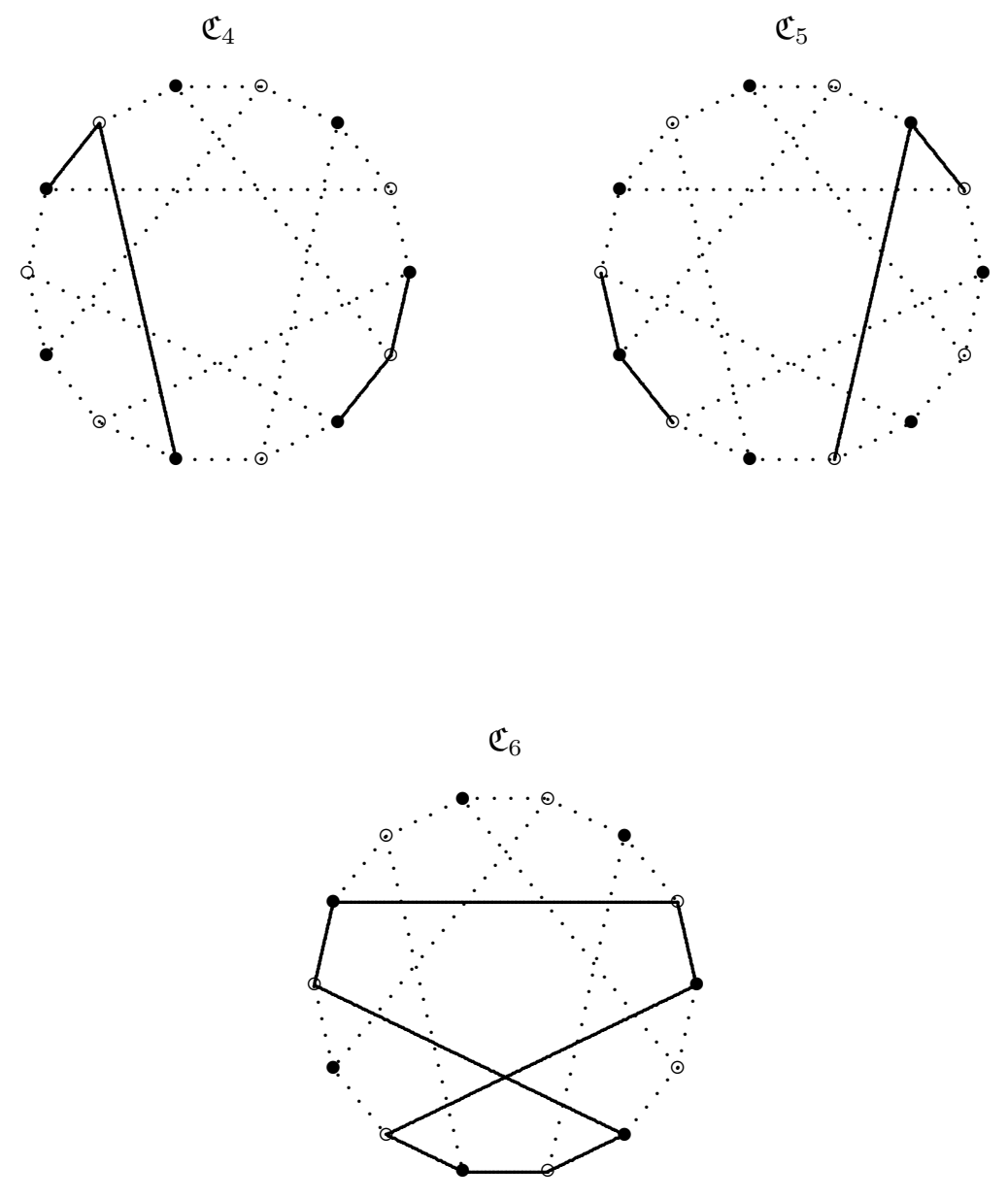

Then $X_{1}=\overline{\mathfrak{c}}_{0}$ and $X_{6}=X_{\infty}$, and the sets $X_{i}$ for $2 \leq i \leq 5$ are given in the following diagrams:
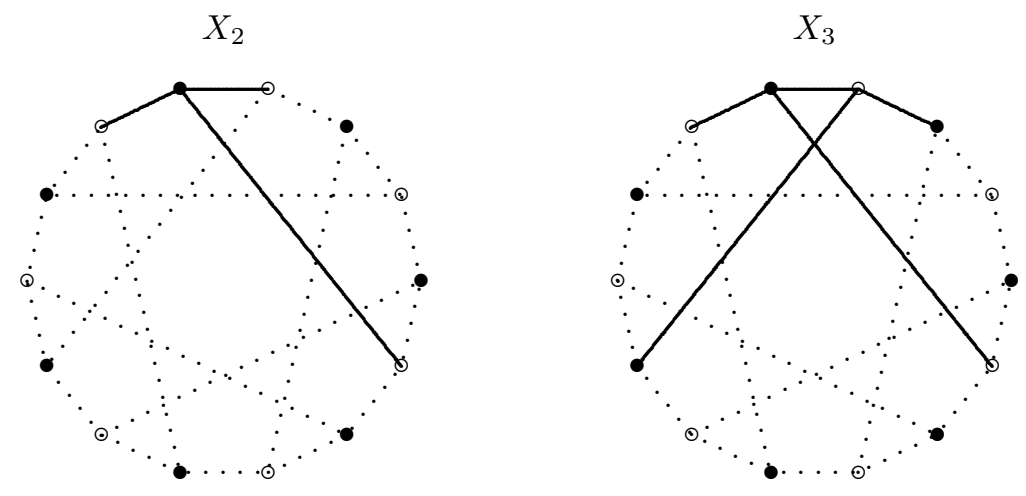

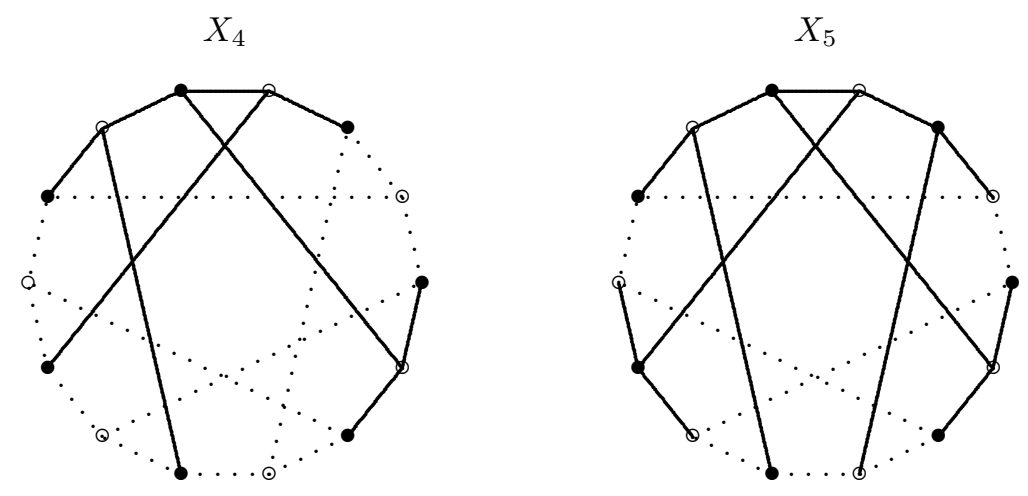

Observe that for $2 \leq m \leq 6$ the set $X_{m}-X_{m-1}$ is a disjoint union of subspaces homeomorphic to $L_{m}$, with one copy of $L_{m}$ for each chamber $\mathfrak{c} \in \mathfrak{C}_{m}$. This is a general phenomenon, as is shown in Proposition 4.5 below.

In order to obtain a general description of the spaces $X_{m}-X_{m-1}$, we begin by defining a topology on the set $\mathcal{S}$ of all sectors in $\Delta$ based at $x_{0}$. The topology has a basis given by sets of the form

$$
N(\sigma, r)=\left\{\sigma^{\prime} \in \mathcal{S} \mid \sigma^{\prime} \cap \bar{B}_{r}=\sigma \cap \bar{B}_{r}\right\}
$$

for $\sigma \in \mathcal{S}$ and $r>0$. Because $\mathcal{S}$ can be identified with the collection $\mathfrak{C}$ of all chambers in $\Delta_{\infty}$, this definition also provides a topology on $\mathfrak{C}$ and on the subspaces $\mathfrak{C}_{i} \subseteq \mathfrak{C}$ for $1 \leq i \leq N$. If $\mathfrak{c} \in \mathfrak{C}$ is a chamber represented by the sector $\sigma \in \mathcal{S}$, then we write $N(\mathfrak{c}, r)$ for the basic open neighborhood of $\mathfrak{c}$ consisting of all chambers represented by sectors in $N(\sigma, r)$. The topological space $\mathcal{S}$ has previously been used by analysts, who usually denote it by $\Omega$ and call it the boundary of the building $\Delta$ (for example, see [MZ] or $[\mathrm{RR}]$ ).

Proposition 4.3. The space $\mathcal{S}$ is compact and totally disconnected. Moreover, $\mathfrak{C}_{i}$ is compact and totally disconnected for $1 \leq i \leq N$.

Proof. The proof of the second statement is virtually identical to that of the first, so we prove the result only for $\mathcal{S}$. Let $\sigma_{1}$ and $\sigma_{2}$ be distinct sectors in $\mathcal{S}$. Then there is an $r>0$ such that $\sigma_{1} \cap \bar{B}_{r} \neq \sigma_{2} \cap \bar{B}_{r}$. Since $\Delta$ is locally finite, $\left\{\sigma_{1}, \sigma_{2}\right\}$ can be extended to a finite collection of sectors $\left\{\sigma_{1}, \ldots, \sigma_{t}\right\}$ such that if $\sigma \in \mathcal{S}$, then there is a unique $i$ with $1 \leq i \leq t$ satisfying $\sigma \cap \bar{B}_{r}=\sigma_{i} \cap \bar{B}_{r}$. Set $U_{1}=N\left(\sigma_{1}, r\right)$ and $U_{2}=N\left(\sigma_{2}, r\right) \cup \cdots \cup N\left(\sigma_{t}, r\right)$. Then $U_{1}$ and $U_{2}$ are disjoint open subsets of $\mathcal{S}$ such that $U_{1} \cup U_{2}=\mathcal{S}$. Thus $\sigma_{1}$ and $\sigma_{2}$ lie in different components of $\mathcal{S}$, and $\mathcal{S}$ is totally disconnected.

For each $i \in \mathbb{N}$ let $F_{i}$ be the collection of subsets of $\partial \bar{B}_{i}$ of the form $\sigma \cap \partial \bar{B}_{i}$ for some $\sigma \in \mathcal{S}$. Then $F_{i}$ is a finite set, so it is a compact topological space with the discrete topology. The space $\prod_{i=1}^{\infty} F_{i}$ is again compact, and there is a map $\psi: \mathcal{S} \rightarrow \prod_{i=1}^{\infty} F_{i}$ given by $\psi(\sigma)=\left(\sigma \cap \partial \bar{B}_{i}\right)_{i \in \mathbb{N}}$. It is straightforward to check that $\psi$ maps $\mathcal{S}$ homeomorphically onto a closed subset. Thus $\mathcal{S}$ is compact, as desired.

Proposition 4.4. The sets $X_{m}$ are closed in $X_{\infty}$ for $1 \leq m \leq N$.

Proof. Let $\left[x_{0}, e\right)$ be any point in $X_{\infty}-X_{m}$, and let $\mathcal{R} \subseteq \mathcal{S}$ be the collection of all sectors that represent a chamber $\mathfrak{c}$ in $\Delta_{\infty}$ with $\left[x_{0}, e\right) \in \overline{\mathfrak{c}}$. Such a chamber must 
satisfy $\delta\left(\mathfrak{c}_{0}, \mathfrak{c}\right)=w_{i}$ for some $i>m$. Let $y \neq x_{0}$ be a point on the geodesic $\left[x_{0}, e\right)$, and set $s=d\left(x_{0}, y\right)$. Choose $\varepsilon>0$ so that the open ball $B_{\varepsilon}(y)$ satisfies

$$
B_{\varepsilon}(y) \subseteq \bigcup_{\sigma \in \mathcal{R}} \bar{\sigma} .
$$

Then $U=B_{s}\left(\left[x_{0}, e\right), \varepsilon\right) \cap X_{\infty}$ is an open neighborhood of $\left[x_{0}, e\right)$ in $X_{\infty}$ that is contained in the union of all closed chambers $\overline{\mathfrak{c}}$ with $\left[x_{0}, e\right) \in \overline{\mathfrak{c}}$.

Suppose that $U \cap X_{m} \neq \emptyset$. Then there exist an $i \leq m$ and a chamber $\mathfrak{c}_{i} \in \mathfrak{C}_{i}$ such that $U \cap \overline{\mathfrak{c}}_{i} \neq \emptyset$. But $U$ is open, so $U \cap \mathfrak{c}_{i} \neq \emptyset$. Since $U$ is contained in the union of all closed chambers $\overline{\mathfrak{c}}$ with $\left[x_{0}, e\right) \in \overline{\mathfrak{c}}$, we obtain a contradiction. Hence $U \cap X_{m}=\emptyset$. It follows that $X_{\infty}-X_{m}$ is open so that $X_{m}$ is closed, as desired.

Proposition 4.5. If $1<m \leq N$, then $X_{m}-X_{m-1}$ is homeomorphic to $\mathfrak{C}_{m} \times L_{m}$.

Proof. For any chamber $\mathfrak{c} \in \mathfrak{C}$ let $\phi_{\mathfrak{c}_{0} \mathfrak{c}}: \overline{\mathfrak{c}}_{0} \rightarrow \overline{\mathfrak{c}}$ be the geometric realization of the unique type-preserving simplicial map from $\mathfrak{c}_{0}$ to $\mathfrak{c}$. If $\mathfrak{c} \in \mathfrak{C}_{m}$, then $\phi_{\mathfrak{c}_{0} \mathfrak{c}}$ restricts to a homeomorphism between $L_{m}$ and $\left(X_{m}-X_{m-1}\right) \cap \overline{\mathfrak{c}}$. Define $\Phi: \mathfrak{C}_{m} \times L_{m} \rightarrow$ $X_{m}-X_{m-1}$ by

$$
\Phi(\mathfrak{c}, \gamma)=\phi_{\mathfrak{c}_{0} \mathfrak{c}}(\gamma)
$$

We claim that $\Phi$ is a homeomorphism.

If $\gamma \in X_{m}-X_{m-1}$, then $\gamma \in \overline{\mathfrak{c}}$ for some chamber $\mathfrak{c} \in \mathfrak{C}_{m}$, and $\gamma \notin \overline{\mathfrak{d}}$ for any chamber $\mathfrak{d} \in \mathfrak{C}_{i}$ with $i<m$. Thus there is a $\gamma_{0} \in L_{m}$ with $\gamma=\phi_{\mathfrak{c}_{0} \mathfrak{c}}\left(\gamma_{0}\right)=\Phi\left(\mathfrak{c}, \gamma_{0}\right)$, and $\Phi$ is onto. Moreover, if $\gamma=\Phi\left(\mathfrak{c}, \gamma_{0}\right)=\Phi\left(\mathfrak{c}^{\prime}, \gamma_{0}^{\prime}\right)$, then $\gamma \in \overline{\mathfrak{c}} \cap \overline{\mathfrak{c}}^{\prime}$. Let $\mathfrak{b}$ be a common face of $\mathfrak{c}$ and $\mathfrak{c}^{\prime}$, and let $\mathfrak{a}_{0}$ be an apartment in $\Delta_{\infty}$ containing $\mathfrak{c}_{0}$ and $\mathfrak{b}$. Because $\gamma \notin X_{m-1}$, any minimal gallery from $\mathfrak{c}_{0}$ to $\mathfrak{c}$ is also a minimal gallery stretched from $\mathfrak{c}_{0}$ to $\mathfrak{b}$, so it is contained in $\mathfrak{a}_{0}$. In particular, $\mathfrak{c}$ is a chamber in $\mathfrak{a}_{0}$. The same argument shows that $\mathfrak{c}^{\prime}$ is also in $\mathfrak{a}_{0}$, and $\delta\left(\mathfrak{c}_{0}, \mathfrak{c}\right)=\delta\left(\mathfrak{c}_{0}, \mathfrak{c}^{\prime}\right)=w_{m}$. Then $\mathfrak{c}=\mathfrak{c}^{\prime}$ and $\gamma_{0}=\gamma_{0}^{\prime}$, so $\Phi$ is a one-to-one correspondence.

Before proving that $\Phi$ and $\Phi^{-1}$ are continuous, it is useful to make a preliminary observation: if $\gamma \in \overline{\mathfrak{c}}_{0}, \mathfrak{c}$ and $\mathfrak{c}^{\prime}$ are chambers of $\Delta_{\infty}$ with $\mathfrak{c}^{\prime} \in N(\mathfrak{c}, r)$, and $y$ is the point on the geodesic $\phi_{\mathfrak{c}_{0} \mathfrak{c}}(\gamma) \in X_{\infty}$ with $d\left(x_{0}, y\right)=r$, then $y$ also lies on the geodesic $\phi_{\mathfrak{c}_{0} \mathfrak{c}^{\prime}}(\gamma)$. Indeed, let $\sigma \in \mathcal{S}$ be the sector corresponding to $\mathfrak{c}$, and let $\sigma^{\prime} \in \mathcal{S}$ be the sector corresponding to $\mathfrak{c}^{\prime}$. Choose apartments $A$ and $A^{\prime}$ in $\Delta$ with $\bar{\sigma} \subseteq A$ and $\bar{\sigma}^{\prime} \subseteq A^{\prime}$. There is a unique type-preserving isometry $\psi: A \rightarrow A^{\prime}$ fixing $A \cap A^{\prime}$ pointwise, and $\psi$ induces a map $\psi_{\infty}: A_{\infty} \rightarrow A_{\infty}^{\prime}$ on the corresponding apartments in $\Delta_{\infty}$. This map satisfies $\psi_{\infty}(\mathfrak{c})=\mathfrak{c}^{\prime}$ because $\sigma^{\prime} \cap \bar{B}_{r}=\sigma \cap \bar{B}_{r}$. Thus $\psi_{\infty} \mid \overline{\mathfrak{c}}=\phi_{\mathfrak{c}_{0} \mathfrak{c}^{\prime}} \circ \phi_{\mathfrak{c}_{0} \mathfrak{c}}^{-1}$, and $\phi_{\mathfrak{c}_{0} \mathfrak{c}^{\prime}}(\gamma)=\psi_{\infty} \phi_{\mathfrak{c}_{0} \mathfrak{c}}(\gamma)$. Moreover, $\left[x_{0}, y\right] \subseteq A \cap A^{\prime}$, so $\psi$ fixes $\left[x_{0}, y\right]$. It follows that $\left[x_{0}, y\right]$ is the initial segment of the geodesics $\phi_{\mathfrak{c}_{0} \mathfrak{c}}(\gamma)$ and $\phi_{\mathfrak{c}_{0} \mathfrak{c}^{\prime}}(\gamma)$, as claimed.

To prove that $\Phi$ is continuous, let $B_{r}\left(\gamma_{0}, \varepsilon\right)$ be a basic open set in $\bar{X}$, and set

$$
\hat{B}_{r}\left(\gamma_{0}, \varepsilon\right)=B_{r}\left(\gamma_{0}, \varepsilon\right) \cap\left(X_{m}-X_{m-1}\right) .
$$

It suffices to show that $\Phi^{-1}\left(\hat{B}_{r}\left(\gamma_{0}, \varepsilon\right)\right)$ is open in $\mathfrak{C}_{m} \times L_{m}$. Suppose that $(\mathfrak{c}, \gamma) \in$ $\Phi^{-1}\left(\hat{B}_{r}\left(\gamma_{0}, \varepsilon\right)\right)$. Define an open subset $V \subseteq L_{m}$ by setting $V=\phi_{\mathfrak{c}_{0} \mathfrak{c}}^{-1}\left(\hat{B}_{r}\left(\gamma_{0}, \varepsilon\right)\right)$. Then $\left(N(\mathfrak{c}, r) \cap \mathfrak{C}_{m}\right) \times V$ is an open neighborhood of $(\mathfrak{c}, \gamma)$ in $\mathfrak{C}_{m} \times L_{m}$, and we claim that it is contained in $\Phi^{-1}\left(\hat{B}_{r}\left(\gamma_{0}, \varepsilon\right)\right)$. Indeed, suppose that $\left(\mathfrak{c}^{\prime}, \gamma^{\prime}\right) \in(N(\mathfrak{c}, r) \cap$ $\left.\mathfrak{C}_{m}\right) \times V$, and let $y \in \Delta$ be the point on the geodesic $\phi_{\mathfrak{c}_{0} \mathfrak{c}}\left(\gamma^{\prime}\right) \in X_{\infty}$ with $d\left(x_{0}, y\right)=r$. Then $\left[x_{0}, y\right]$ is also the initial segment of the geodesic $\phi_{\mathfrak{c}_{0} \mathfrak{c}^{\prime}}\left(\gamma^{\prime}\right)$. Because $\gamma^{\prime} \in V$, we have $d\left(\gamma_{0}(r), y\right)<\varepsilon$. Then $\Phi\left(\mathfrak{c}^{\prime}, \gamma^{\prime}\right)=\phi_{\mathfrak{c}_{0} \mathfrak{c}^{\prime}}\left(\gamma^{\prime}\right) \in \hat{B}_{r}\left(\gamma_{0}, \varepsilon\right)$, as desired. 
Finally, we must show that $\Phi$ maps open subsets of $\mathfrak{C}_{m} \times L_{m}$ to open subsets of $X_{m}-X_{m-1}$. It suffices to prove this result for an open set of the form $N(\mathfrak{c}, r) \times$ $\left(B_{r^{\prime}}(\gamma, \varepsilon) \cap L_{m}\right)$ with $\mathfrak{c} \in \mathfrak{C}_{m}$ and $\gamma \in L_{m}$. Note that the image of this set can be written as a disjoint union:

$$
\begin{aligned}
\Phi\left(N(\mathfrak{c}, r) \times\left(B_{r^{\prime}}(\gamma, \varepsilon) \cap L_{m}\right)\right) & =\bigcup_{\mathfrak{c}^{\prime} \in N(\mathfrak{c}, r)} \phi_{\mathfrak{c}_{0} \mathfrak{c}^{\prime}}\left(B_{r^{\prime}}(\gamma, \varepsilon) \cap L_{m}\right) \\
& =\bigcup_{\mathfrak{c}^{\prime} \in N(\mathfrak{c}, r)} B_{r^{\prime}}\left(\phi_{\mathfrak{c}_{0} \mathfrak{c}^{\prime}}(\gamma), \varepsilon\right) \cap \phi_{\mathfrak{c}_{0} \mathfrak{c}^{\prime}}\left(L_{m}\right) \\
& =\bigcup_{\mathfrak{c}^{\prime} \in N(\mathfrak{c}, r)} B_{r^{\prime}}\left(\phi_{\mathfrak{c}_{0} \mathfrak{c}^{\prime}}(\gamma), \varepsilon\right) \cap\left(X_{m}-X_{m-1}\right) \cap \overline{\mathfrak{c}}^{\prime}
\end{aligned}
$$

Assume that $r^{\prime} \leq r$. Then $B_{r^{\prime}}\left(\phi_{\mathfrak{c}_{0} \mathfrak{c}^{\prime}}(\gamma), \varepsilon\right)=B_{r^{\prime}}\left(\phi_{\mathfrak{c}_{0} \mathfrak{c}}(\gamma), \varepsilon\right)$ for all $\mathfrak{c}^{\prime} \in N(\mathfrak{c}, r) \subseteq$ $N\left(\mathfrak{c}, r^{\prime}\right)$, so

$$
\begin{aligned}
\Phi\left(N(\mathfrak{c}, r) \times\left(B_{r^{\prime}}(\gamma, \varepsilon) \cap L_{m}\right)\right) & =B_{r^{\prime}}\left(\phi_{\mathfrak{c}_{0} \mathfrak{c}}(\gamma), \varepsilon\right) \cap \bigcup_{\mathfrak{c}^{\prime} \in N(\mathfrak{c}, r)}\left(X_{m}-X_{m-1}\right) \cap \overline{\mathfrak{c}}^{\prime} \\
& =B_{r^{\prime}}\left(\phi_{\mathfrak{c}_{0} \mathfrak{c}}(\gamma), \varepsilon\right) \cap \Phi\left(N(\mathfrak{c}, r) \times L_{m}\right)
\end{aligned}
$$

and it suffices to prove that $\Phi\left(N(\mathfrak{c}, r) \times L_{m}\right)$ is open. Suppose that $\gamma^{\prime} \in\left(X_{m}-\right.$ $\left.X_{m-1}\right) \cap \overline{\mathfrak{c}}^{\prime}$ for some $\mathfrak{c}^{\prime} \in N(\mathfrak{c}, r)$. If there is a chamber $\mathfrak{c}^{\prime \prime} \neq \mathfrak{c}^{\prime}$ such that $\gamma^{\prime} \in \overline{\mathfrak{c}}^{\prime \prime}$, then $\delta\left(\mathfrak{c}_{0}, \mathfrak{c}^{\prime \prime}\right)=w_{k}$ for some $k \geq m$ because $\gamma^{\prime} \in X_{m}-X_{m-1}$; in fact, $k>m$ because $\Phi$ is one-to-one. If there is no such chamber $\mathfrak{c}^{\prime \prime}$, then $\gamma^{\prime}$ lies in the interior $\mathfrak{c}^{\prime}$ of $\overline{\mathfrak{c}}^{\prime}$. In either case there is a number $\varepsilon^{\prime}>0$ such that

$$
B_{r}\left(\gamma^{\prime}, \varepsilon^{\prime}\right) \cap X_{\infty} \subseteq\left(\bigcup_{\mathfrak{c}^{\prime} \in N(\mathfrak{c}, r)} \overline{\mathfrak{c}}^{\prime}\right) \cup\left(\bigcup_{k=m+1}^{N} \bigcup_{\mathfrak{c}^{\prime \prime} \in \mathfrak{C}_{k}} \overline{\mathfrak{c}}^{\prime \prime}\right) .
$$

Then $\gamma^{\prime} \in B_{r}\left(\gamma^{\prime}, \varepsilon^{\prime}\right) \cap\left(X_{m}-X_{m-1}\right) \subseteq \Phi\left(N(\mathfrak{c}, r) \times L_{m}\right)$, so $\Phi\left(N(\mathfrak{c}, r) \times L_{m}\right)$ is open in $X_{m}-X_{m-1}$, as desired.

Now suppose that $r<r^{\prime}$, and let $\sigma \in \mathcal{S}$ be the sector corresponding to the chamber $\mathfrak{c}$. Because $\Delta$ is locally finite, there exists a finite set of sectors $\left\{\sigma_{1}, \ldots, \sigma_{t}\right\} \subseteq$ $N(\sigma, r)$ such that if $\sigma^{\prime} \in N(\sigma, r)$, then $\sigma^{\prime} \cap \bar{B}_{r^{\prime}}=\sigma_{i} \cap \bar{B}_{r^{\prime}}$ for a unique $i$ with $1 \leq i \leq t$. Let $\mathfrak{c}_{i} \in N(\mathfrak{c}, r)$ be the chamber corresponding to $\sigma_{i}$. Then

$$
\begin{aligned}
\Phi\left(N(\mathfrak{c}, r) \times\left(B_{r^{\prime}}(\gamma, \varepsilon) \cap L_{m}\right)\right) & =\bigcup_{i=1}^{t} \bigcup_{\mathfrak{c}^{\prime} \in N\left(\mathfrak{c}_{i}, r^{\prime}\right)} B_{r^{\prime}}\left(\phi_{\mathfrak{c}_{0} \mathfrak{c}^{\prime}}(\gamma), \varepsilon\right) \cap\left(X_{m}-X_{m-1}\right) \cap \overline{\mathfrak{c}}^{\prime} \\
& =\bigcup_{i=1}^{t} \Phi\left(N\left(\mathfrak{c}_{i}, r^{\prime}\right) \times\left(B_{r^{\prime}}(\gamma, \varepsilon) \cap L_{m}\right)\right),
\end{aligned}
$$

and this set is open in $X_{m}-X_{m-1}$ by the previous paragraph. Thus $\Phi$ is a homeomorphism, and this completes the proof.

We are now able to calculate the Alexander-Spanier cohomology $H^{*}\left(X_{\infty} ; \mathbb{Z}\right)$.

Theorem 4.6. Let $n=\operatorname{dim} \Delta$. Then the reduced cohomology $\tilde{H}^{i}\left(X_{\infty} ; \mathbb{Z}\right)$ is 0 for $i \neq n-1$ and is a free abelian group for $i=n-1$.

Proof. For $1<m<N$ let $L_{m}^{\prime}$ be the complement of $L_{m}$ in $\overline{\mathfrak{c}}_{0}$. Then $L_{m}^{\prime}$ is the union of the closures of the faces $\left(\mathfrak{c}_{0}\right)_{\{s\}}$ of codimension one for $s \in S-I_{m}$. Since $I_{m} \neq \emptyset$ 
and $I_{m} \neq S$, the subspace $L_{m}^{\prime}$ is contractible. The exact cohomology sequence

$$
\cdots \rightarrow \tilde{H}^{i-1}\left(L_{m}^{\prime} ; \mathbb{Z}\right) \rightarrow H_{c}^{i}\left(L_{m} ; \mathbb{Z}\right) \rightarrow \tilde{H}^{i}\left(\overline{\mathfrak{c}}_{0} ; \mathbb{Z}\right) \rightarrow \tilde{H}^{i}\left(L_{m}^{\prime} ; \mathbb{Z}\right) \rightarrow \cdots
$$

implies that $H_{c}^{i}\left(L_{m} ; \mathbb{Z}\right)=0$ for all $i$. By Proposition 4.5 and the Künneth Theorem it follows that $H_{c}^{*}\left(X_{m}-X_{m-1} ; \mathbb{Z}\right)=0$.

The space $X_{1}=\overline{\mathfrak{c}}_{0}$ is contractible, so its reduced cohomology is zero. Applying the long exact cohomology sequence inductively to the pairs $\left(X_{m}, X_{m-1}\right)$, we conclude that $H^{*}\left(X_{m} ; \mathbb{Z}\right)=0$ for $m<N$ and that

$$
\tilde{H}^{*}\left(X_{\infty} ; \mathbb{Z}\right) \cong H_{c}^{*}\left(X_{\infty}-X_{N-1} ; \mathbb{Z}\right) .
$$

By Proposition 4.5 there is a homeomorphism between $X_{\infty}-X_{N-1}$ and $\mathfrak{C}_{N} \times L_{N}=$ $\mathfrak{C}_{N} \times \mathfrak{c}_{0}$. The space $\mathfrak{c}_{0}$ is homeomorphic to $\mathbb{R}^{n-1}$, so

$$
H_{c}^{i}\left(\mathfrak{c}_{0} ; \mathbb{Z}\right)= \begin{cases}\mathbb{Z} & \text { if } i=n-1 \\ 0 & \text { otherwise }\end{cases}
$$

Thus the Künneth Theorem implies that

$$
H_{c}^{i}\left(X_{\infty}-X_{N-1} ; \mathbb{Z}\right) \cong H_{c}^{i-n+1}\left(\mathfrak{C}_{N} ; \mathbb{Z}\right)
$$

Because $\mathfrak{C}_{N}$ is totally disconnected, $H_{c}^{i}\left(\mathfrak{C}_{N} ; \mathbb{Z}\right)=0$ for $i>0$ so that $\tilde{H}^{i}\left(X_{\infty} ; \mathbb{Z}\right)=0$ for $i \neq n-1$. Moreover, $H_{c}^{0}\left(\mathfrak{C}_{N} ; \mathbb{Z}\right)$ is a free abelian group because $\mathfrak{C}_{N}$ is a locally compact Hausdorff space (see Section 4.4 of $[\mathrm{Ma}]$ ). Thus $\tilde{H}^{n-1}\left(X_{\infty} ; \mathbb{Z}\right)$ is free abelian, and this completes the proof.

Corollary 4.7. Let $n=\operatorname{dim} \Delta$. Then the cohomology $H_{c}^{i}(\Delta ; \mathbb{Z})$ is 0 for $i \neq n$ and is a free abelian group for $i=n$.

Proof. The space $\Delta$ is homeomorphic to $X$, which is an open subspace of the contractible space $\bar{X}$. Since $X_{\infty}=\bar{X}-X$ is closed and $\bar{X}$ is compact, the exact cohomology sequence

$$
\cdots \rightarrow \tilde{H}^{i-1}(\bar{X} ; \mathbb{Z}) \rightarrow \tilde{H}^{i-1}\left(X_{\infty} ; \mathbb{Z}\right) \rightarrow H_{c}^{i}(X ; \mathbb{Z}) \rightarrow \tilde{H}^{i}(\bar{X} ; \mathbb{Z}) \rightarrow \cdots
$$

implies that $H_{c}^{i}(\Delta ; \mathbb{Z}) \cong H_{c}^{i}(X ; \mathbb{Z}) \cong \tilde{H}^{i-1}\left(X_{\infty} ; \mathbb{Z}\right)$ for all $i$. The desired result now follows from Theorem 4.6

\section{Finiteness PROPERTIES OF $\widetilde{A}_{n}$-GROUPS}

In this section we prove that finitely presented $\widetilde{A}_{n}$-groups satisfy a number of other finiteness properties. In particular, these groups are virtually torsion-free and have finite virtual cohomological dimension. The results of the previous section imply that they are virtual duality groups, and we end by showing that they have only finitely many conjugacy classes of finite subgroups.

A group $\Gamma$ is an $\widetilde{A}_{n}$-group if it acts simply transitively on the vertices of a thick $\widetilde{A}_{n}$-building in a type-rotating manner. By results of [CMSZ1] and [CMSZ2] for $n=2$ and of $[\mathrm{C}$ for $n>2$, it is known that $\Gamma$ has a simple presentation in terms of a projective geometry. In particular, suppose that $\Pi$ is an $n$-dimensional projective geometry, and let $\Pi_{i}$ denote the set of elements of dimension $i$ in $\Pi$. Suppose that $\lambda: \Pi \rightarrow \Pi$ is an involution satisfying $\lambda\left(\Pi_{i}\right)=\Pi_{n+1-i}$ for $1 \leq i \leq n$. A set $\mathcal{T}$ of ordered triples of elements in $\Pi$ is called an $\widetilde{A}_{n}$ triangle presentation compatible with $\lambda$ if it satisfies the following conditions:

(1) Let $u, v \in \Pi$. Then there is a $w \in \Pi$ such that $(u, v, w) \in \mathcal{T}$ if and only if $\lambda(u)$ and $v$ are distinct and non-incident. 
(2) If $(u, v, w) \in \mathcal{T}$, then $(v, w, u) \in \mathcal{T}$.

(3) If $(u, v, w) \in \mathcal{T}$ and $\left(u, v, w^{\prime}\right) \in \mathcal{T}$, then $w=w^{\prime}$.

(4) If $(u, v, w) \in \mathcal{T}$, then $(\lambda(w), \lambda(v), \lambda(u)) \in \mathcal{T}$.

(5) If $(u, v, w) \in \mathcal{T}$, then $\operatorname{dim} u+\operatorname{dim} v+\operatorname{dim} w \equiv 0(\bmod n+1)$.

(6) For $i=1,2$ set $\mathcal{T}^{i}=\{(u, v, w) \in \mathcal{T} \mid \operatorname{dim} u+\operatorname{dim} v+\operatorname{dim} w=i(n+1)\}$. If $(u, v, w) \in \mathcal{T}^{2}$ and $\left(u, v^{\prime}, w^{\prime}\right) \in \mathcal{T}^{1}$, then there exists an element $x \in \Pi$ such that $\left(\lambda(v), v^{\prime}, x\right) \in \mathcal{T}^{1}$ and $\left(w, \lambda\left(w^{\prime}\right), x\right) \in \mathcal{T}^{2}$.

If $\mathcal{T}$ is an $\widetilde{A}_{n}$ triangle presentation, then there is an associated group $\Gamma_{\mathcal{T}}$ generated by the symbols $a_{u}$ for $u \in \Pi$ and satisfying the relations $a_{u} a_{\lambda(u)}=1$ for $u \in \Pi$ and $a_{u} a_{v} a_{w}=1$ for $(u, v, w) \in \mathcal{T}$. The results of [CMSZ1, CMSZ2], and [C] show that the Cayley graph of $\Gamma_{\mathcal{T}}$ with respect to this presentation is the 1-skeleton of a thick $\widetilde{A}_{n}$-building $\Delta_{\mathcal{T}}$. In particular, $\Gamma_{\mathcal{T}}$ acts simply transitively on the vertices of $\Delta_{\mathcal{T}}$ in a type-rotating manner, so it is an $\widetilde{A}_{n}$-group. Conversely, every $\widetilde{A}_{n}$-group is isomorphic to $\Gamma_{\mathcal{T}}$ for some $\widetilde{A}_{n}$ triangle presentation $\mathcal{T}$.

For the remainder of this section we assume that $\mathcal{T}$ is an $\widetilde{A}_{n}$ triangle presentation as above. Set $\Gamma=\Gamma_{\mathcal{T}}$ and $\Delta=\Delta_{\mathcal{T}}$. Since $\operatorname{dim} u+\operatorname{dim} v+\operatorname{dim} w \equiv 0(\bmod n+1)$ for all $(u, v, w) \in \mathcal{T}$ and $\operatorname{dim} \lambda(u)=n+1-\operatorname{dim} u$ for all $u \in \Pi$, the map $\tau: \Gamma \rightarrow \mathbb{Z}_{n+1}$ defined on generators of $\Gamma$ by $\tau\left(a_{u}\right)=\operatorname{dim} u$ extends to a group epimorphism. We begin by studying the properties of the subgroup $K=\operatorname{Ker} \tau$ and of the stabilizer $\Gamma_{\sigma}$ of a simplex $\sigma$ in $\Delta$.

Proposition 5.1. Let $\sigma$ be any simplex in $\Delta$. If $\gamma \in \Gamma_{\sigma}-\{1\}$, then $\gamma$ is conjugate to a generator of $\Gamma$ of order dividing $n+1$. Moreover, $K_{\sigma}=1$.

Proof. Identify the vertices of $\Delta$ with the elements of $\Gamma$ and the 1-skeleton with the Cayley graph. Because $\gamma \neq 1$, no vertex of $\sigma$ is fixed by $\gamma$. In particular, if $v$ is a vertex of $\sigma$, then $\gamma v \neq v$ is also a vertex of $\sigma$ because $\gamma \in \Gamma_{\sigma}$. Thus $\gamma v=v a_{u}$ for some generator $a_{u}$ of $\Gamma$. Since $\gamma$ permutes the vertices of $\sigma$, we have $\gamma^{m} v=v$ for some $m>0$. Thus $\gamma^{m}=1$, and $a_{u}=v^{-1} \gamma v$ is a generator of finite order.

If $\gamma^{\prime} \in K_{\sigma}-\{1\}$, then $\gamma^{\prime}$ is conjugate to some generator $a_{u^{\prime}}$. But then

$$
\operatorname{dim} u^{\prime}=\tau\left(a_{u^{\prime}}\right) \equiv \tau\left(\gamma^{\prime}\right) \equiv 0 \quad(\bmod n+1),
$$

and we have a contradiction. Hence $K_{\sigma}=1$. Since $K$ is normal in $\Gamma$ and $|\Gamma: K|=$ $n+1$, it follows that $\gamma^{n+1} \in K \cap \Gamma_{\sigma}=1$. Thus $\gamma$ is conjugate to a generator of order dividing $n+1$, and this completes the proof.

Proposition 5.2. Let $H$ be a subgroup of $\Gamma$. Then $H$ is finite if and only if $H$ stabilizes some simplex $\sigma$ of $\Delta$. In particular, $\Gamma_{\sigma}$ is cyclic of order dividing $n+1$ for every simplex $\sigma$ of $\Delta$.

Proof. Assume that $H$ is finite, and let $x$ be any point in $\Delta$. Then the orbit $H x=$ $\{h \cdot x \mid h \in H\}$ is bounded. By the Bruhat-Tits Fixed Point Theorem there is a point $y \in \Delta$ that is fixed by $H$. Let $\sigma$ be the unique simplex of minimum dimension containing $y$. Then $H$ stabilizes $\sigma$.

To prove the remainder of the proposition, it is only necessary to observe that if $\sigma$ is any simplex of $\Delta$, then $\Gamma_{\sigma} \cong \Gamma_{\sigma} K / K$ is a subgroup of $\Gamma / K \cong \mathbb{Z}_{n+1}$. Thus $\Gamma_{\sigma}$ is cyclic of order dividing $n+1$, as claimed.

Propositions 5.1 and 5.2 show that any element $\gamma \in \Gamma-\{1\}$ of finite order is conjugate to a generator of order dividing $n+1$. The proof given here was previously known to D. Cartwright, who has also found a purely algebraic proof for $\widetilde{A}_{2}$-groups. 
The remaining results are proven under the assumption that the projective geometry $\Pi$ is finite. This condition implies that $\Gamma$ is finitely generated and that the building $\Delta$ is locally finite so that the results of the previous sections are applicable.

Lemma 5.3. Assume that the projective geometry $\Pi$ is finite. Then $\Delta$ has finitely many simplices modulo $K$.

Proof. Let $v$ be any 0 -simplex in $\Delta$. Since $\Pi$ is finite, there are only finitely many simplices $\sigma_{1}, \ldots, \sigma_{s}$ having $v$ as a vertex. Let $\sigma^{\prime}$ be any simplex of $\Delta$, and let $v^{\prime}$ be a vertex of $\sigma^{\prime}$. Then there is an element $\gamma \in \Gamma$ with $\gamma v^{\prime}=v$, so $\gamma \sigma^{\prime}=\sigma_{i}$ for some $i$ with $1 \leq i \leq s$. Thus $\Delta$ has only finitely many simplices modulo $\Gamma$. Since $|\Gamma: K|=n+1$, it follows that $\Delta$ also has finitely many simplices modulo $K$, as desired.

A group $K$ is said to be of type $F P$ if $\mathbb{Z}$ admits a projective resolution over $\mathbb{Z} K$ that has finite length and consists of finitely generated modules. If there is a largest integer $d \geq 0$ such that $H^{d}(K, M) \neq 0$ for some $\mathbb{Z} K$-module $M$, then $K$ is said to have cohomological dimension $\mathrm{cd} K=d$.

Proposition 5.4. Assume that the projective geometry $\Pi$ is finite. Then $K \subseteq \Gamma$ is a torsion-free subgroup of type $F P$, and $\operatorname{cd} K \leq n$.

Proof. The building $\Delta$ is contractible, and it is a free $K$-complex by Proposition 5.1 Thus the augmented cellular chain complex $C_{*}(\Delta)$ of $\Delta$ is a free resolution of $\mathbb{Z}$ over $\mathbb{Z} K$ by Proposition I.4.1 of [B1]. Since $\Delta$ has finitely many simplices modulo $K$, it follows that $C_{m}(\Delta)$ is a finitely generated $\mathbb{Z} K$-module for all $m$. Hence $K$ is of type FP. Moreover, cd $K \leq n$ because $C_{m}(\Delta)=0$ for $m>n$, and Corollary VIII.2.5 of [B1] implies that $K$ is torsion-free.

Theorem 5.5. Assume that the projective geometry $\Pi$ is finite. Then $\Gamma$ is a virtual duality group.

Proof. The building $\Delta$ is a contractible $K$-complex with only finitely many cells modulo $K$, and $K_{\sigma}=1$ for every simplex $\sigma$. These conditions imply $H^{*}(K, \mathbb{Z} K) \cong$ $H_{c}^{*}(\Delta ; \mathbb{Z})$ (for example, see [B1], p. 209, Exercise 4). Moreover, $H_{c}^{n}(\Delta ; \mathbb{Z})$ is torsionfree, and $H_{c}^{i}(\Delta ; \mathbb{Z})=0$ for $i \neq n$. Since $K$ is of type FP, Theorem VIII.10.1 of [B1] implies that it is a duality group, and the desired result follows.

In many cases it is possible to give a shorter proof of Theorem [5.5. For example, suppose that $n>2$. Then $\Gamma$ is an $S$-arithmetic group by Corollary 10.25 of $[\mathbb{R}$ and Margulis' Arithmeticity Theorem (Theorem 10.1.12 of [Z]). Hence $K$ is torsion-free and $S$-arithmetic, so it is a duality group by $\mathrm{BS}$.

Now suppose that $n=2$ and that the projective plane $\Pi$ is desarguesian. Then $\Gamma$ has Kazhdan's Property (T) by [CMS], and Corollary III.2.15 of [M] implies that $K$ also has Property (T) because $|\Gamma: K|$ is finite. Thus $K$ does not decompose as a free product by Theorem III.3.9 of [M]. Since cd $K \leq 2$, it follows that $K$ is a duality group (see [B1], p. 185, Examples 1 and 2, and p. 223, Remark 5). The authors are grateful to $\mathrm{G}$. Robertson for pointing out this argument.

Proposition 5.6. Assume that the projective geometry $\Pi$ is finite. Then $\Gamma$ has only finitely many conjugacy classes of finite subgroups.

Proof. If $H$ is a finite subgroup of $\Gamma$, then Proposition 5.2 implies that $H$ is cyclic and stabilizes some simplex of $\Delta$. Let $h$ be a generator of $H$. Then $h$ is conjugate 
to a generator $a_{u} \in \Gamma$ of order dividing $n+1$ by Proposition 5.1. Since $\Pi$ is finite, the number of subgroups $\left\langle a_{u}\right\rangle$ is also finite, and the result follows.

\section{REFERENCES}

[B1] K. S. Brown, Cohomology of Groups, Springer-Verlag, New York, 1982; corrected reprint, 1994. MR 83k:20002 MR 96a:20072

[B2] K. S. Brown, Buildings, Springer-Verlag, New York, 1989; corrected reprint, 1998. MR 90e:20001 MR 99d:20042

[B3] K. S. Brown, Five lectures on buildings; in Group Theory from a Geometrical Viewpoint, E. Ghys, A. Haefliger, and A. Verjovsky, eds., World Sci. Publishing, River Edge, NJ (1991) 254-295. MR 94b:51017

[BS] A. Borel and J.-P. Serre, Cohomologie d'immeubles et de groupes S-arithmétiques, Topology 15 (1976) 211-232. MR 56:5786

[C] D. Cartwright, Groups acting simply transitively on the vertices of a building of type $\widetilde{A}_{n}$; in Groups of Lie Type and their Geometries, London Math. Soc. Lecture Note Ser. 207, Cambridge Univ. Press, Cambridge (1995) 43-76. MR 96a:20039

[CMS] D. Cartwright, W. Młotkowski and T. Steger, Property $(T)$ and $\widetilde{A}_{2}$ groups, Ann. Inst. Fourier 44 (1994) 213-248. MR 95j:20024

[CMSZ1] D. Cartwright, A. M. Mantero, T. Steger, and A. Zappa, Groups acting simply transitively on the vertices of a building of type $\widetilde{A}_{2}$, I, Geom. Dedicata 47 (1993) 143-166. MR 95b:20053

[CMSZ2] D. Cartwright, A. M. Mantero, T. Steger, and A. Zappa, Groups acting simply transitively on the vertices of a building of type $\widetilde{A}_{2}$, II: The cases $q=2$ and $q=3$, Geom. Dedicata 47 (1993) 167-223. MR 95b:20054

[M] G. A. Margulis, Discrete Subgroups of Semisimple Lie Groups, Springer-Verlag, Berlin, 1991. MR 92h:22021

[Ma] W. S. Massey, Homology and Cohomology Theory: An Approach Based on AlexanderSpanier Cochains, Marcel Dekker, New York, 1978. MR 58:7594

[Mu] J. R. Munkres, Topology: A First Course, Prentice-Hall, Englewood Cliffs, NJ, 1975. MR 57:4063

[MZ] A. M. Mantero and A. Zappa, Spherical functions and spectrum of the Laplace operators on buildings of rank 2, Boll. Un. Mat. Ital. B (7) 8 (1994) no. 2, 419-475. MR 95f:43009

[P] F. Paulin, Constructions of hyperbolic groups via hyperbolizations of polyhedra; in Group Theory from a Geometrical Viewpoint, E. Ghys, A. Haefliger, and A. Verjovsky, eds., World Sci. Publishing, River Edge, NJ (1991) 313-372. MR 93d:57005

[R] M. Ronan, Lectures on Buildings, Academic Press, New York, 1989. MR 90j:20001

[RR] J. Ramagge and G. Robertson, Triangle buildings and actions of type $\mathrm{III}_{1 / q^{2}}$, J. Funct. Anal. 140 (1996) 472-504. MR 98b:46089

[S] E. H. Spanier, Algebraic Topology, Springer-Verlag, New York, 1981. MR 83i:55001

[Z] R. J. Zimmer, Ergodic Theory and Semisimple Groups, Monographs in Mathematics 81, Birkhäuser, Boston, 1984. MR 86j:22014

Department of Mathematics, University of Newcastle, NSW 2308 Callaghan, AusTRALIA

E-mail address: jacqui@maths.newcastle.edu.au

Center for Communications Research, 4320 Westerra Court, San Diego, California 92117

E-mail address: wheeler@member.ams.org 